\title{
REVIEW
}

\section{Humanistic Spirit Contained in Traditional Chinese Medicine should Be Valued}

\author{
Qunli Wu, Xiaochun Liang*
}

\author{
Department of Traditional Chinese Medicine, Peking Union Medical \\ College Hospital, Peking Union Medical College \& Chinese \\ Academy of Medical Sciences, Beijing 100730, China
}

\begin{abstract}
Key words: Traditional Chinese Medicine; humanistic spirit; doctor-patient relationship; medical ethics
\end{abstract}

\begin{abstract}
Medicine is a science studying human's health and diseases as well as the regularity between them. Its research subject is human, who possess natural attribute, psychological attribute and social attribute. Therefore, medicine is bound to possess humanistic attribute. Rooted in Chinese traditional culture, Traditional Chinese Medicine contains abundant humanistic thoughts, for instance, pursuing the unity of human and nature, advocating the vital importance of life, sticking to the virtue of "medicine being humane art", abiding by the principle of "Benevolence prior to interest", complying with the medical rule of "Respecting peers". These are the very concrete reflections of medical humanistic spirit. In this article we aim to explore the humanistic thoughts contained in Traditional Chinese Medicine, and try to inherit and carry forward the spirit to better relieve the tensions between doctors and patients and improve the quality of medical services.
\end{abstract}

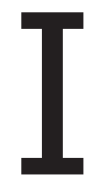

MPACTED by market economy, deficiency in medical health care resources and hysteretic medical health care system, the conflicts between patients and doctors are rapidly worsening. Zhang conducted a large-scale survey crossing several provinces on the status of medical practitioners. It turned out that $74.9 \%$ of medical staffs thought the current doctors-patients relationship in China was in tension. And $35.7 \%$ of the respondents stood in the middle, thinking it neither good nor bad. Only $3.6 \%$ of them felt it is harmonious. ${ }^{[1-2]}$ If such situations get no improvement, there are bound to be

Received for publication September 15, 2018. Published online February 22, 2019.

*Corresponding author E-mail: xcliang@vip.sina.com "great losses of both doctors and patients". That is to say, patients cannot receive the best medical services and doctors cannot improve their medical skills.

Medicine is a science studying human's health and diseases as well as the regularity between them. Its research subject is human who possess three attributes, that is, nature, psychology and society. Therefore, medicine is bound to possess humanistic attribute. Rooted in Chinese traditional culture, Traditional Chinese Medicine contains abundant humanistic thoughts, for instance, pursuing the unity of human and nature, advocating the vital importance of life, sticking to the virtue of "medicine being humane art", abiding by the principle of "Benevolence being prior to interest", complying with the medical rule of "Respecting peers". These are the very concrete re- 
flections of Humanistic Spirit. To build up harmonious doctors-patients relationship we need to carry out the soul of humanism spirit derived from Traditional Chinese Medicine.

\section{The unity of man and nature}

"Humanistic Spirit" first appeared in The Book of Changes. It is said in the divinatory symbols of $\mathrm{Bi}$, "Human beings are born to be either male or female, males are hard and rigid while females are soft and flexible. Rigidness and softness staggered, that is Astronomy, in other words, Nature". Based on this regulation, man and woman pair together to form a couple, then a family, a country, a world. That is Humanism, viz., Culture. Humanistic spirit here refers to humanization, which means educating by civilization. In a general sense, it refers to human civilization. Moreover, it holds that all these only can be created and achieved by having a good handle on the relationship between human and nature. That is "Human are civilized and world become humanized". ${ }^{[3]}$

From the viewpoint of Traditional Chinese Medicine, "human, heaven and earth and everything are integrated as a whole", "The sky supplies people with five $\mathrm{Q} \mathrm{i}$, and the ground supplies people with five tastes". Any changes in the nature, for example, the alternation of seasons, diversity of regions will affect human body directly or indirectly by reflecting different physiological activities or pathological changes. Just as recorded in Lingshu: "striae and interstitial spaces open either when it is too hot or one wears too much, resulting in sweating. Striae and interstitial spaces converge when it is cold, then Qi and fluid cannot move normally and moisture cannot be excreted from the sweat gland but flows to the bladder, transforming into urine". This not only happens along with seasonal alterations, even the changes between day and night in a day bring varied effects to the physiological activities of human body. Human body needs to adapt to these changes. "Yang Qi predominates in the daytime. In the morning, Yang Qi emerges when the sun rises, thrives when the sun is at the high noon, decreases when the sun sets along with the sweat pore closing". Besides, to some extent, diversity of regions and living environment has influence on the physiological activities of human body and the functions of internal organs, causing difference of constitutions. For instance, in the damp and hot south, human's striae and interstitial spaces mostly appear to be sparse; while in the dry and cold north, human's striae and interstitial spaces are mainly dense. Once living in a foreign place, people tend to feel uncomfortable at the beginning, which is called "non-acclimatization". Quite some time needs to be taken for them to get used to it. This is so-called "Correspondence between Man and Nature". However, disease will develop if the climate changes intensely, which is already beyond the human's self-regulation. Or human cannot adjust themselves according to environmental changes due to the disorder of human body's self-regulation. For example, if it is cold when it should be warm in spring, people often catch a cold easily. If it is too hot in summer, people are more likely to suffer from heatstroke. Therefore, in Plain Questions, it pointed out that "YinYang and four seasons are the source and end of everything, the fundamental factor of life and death. If going against the laws of Yin-Yang and four seasons, disasters will occur. But if complying with the rules of Yin-Yang and four seasons, severe deceases won't attack". This emphasizes the idea that "human beings should follow the laws of four seasons and adapt themselves to the climate, calm the mood, regulate Yin-Yang and balance hard stems and soft terms". ${ }^{[4]}$

Correspondingly, treatment based on syndrome differentiation of Traditional Chinese Medicine is to treat in accordance with climate, locality and individuality, that is to say, to adopt different therapies according to different constitutions of the patient, climates and social changes. As the saying goes, "You cannot see the woods for trees". Likewise, cure of disease could not be done without considering all the related factors.

\section{The vital importance of life}

In Plain Questions, it declared that "We have everything in the expanse of heaven and earth, but nothing could be more important than mankind". Traditional Chinese Medicine has constantly strengthened "Putting people first", "Treating a disease and helping a person is the bounden duty of a physician", and "Respecting life is the ultimate truth". Sun Simiao from Tang Dynasty believed, "A man's life is invaluable, even more precious than gold. Saving a person with one prescription is much more valuable than gold". In Plain Questions, it had clearly pointed out the mode of doctor-patient relationship, "Patient is the source. Doctor is the treatment. If the patient fails to cooperate with doctor, the disease cannot be cured". "Patient is the source" refers to the fact that the disease itself and the patient himself are the major aspect. "Doctor is the treatment" 
indicates that doctors and what therapeutic measures taken are the secondary aspect. Only when doctors observe this mode of doctor-patient relationship can they accurately diagnose and obtain good therapeutic results. "Patient is the source" reflects physicians' respect for patients' personality, will and other specific situations. When patients feel and recognize this kind of respect, a favorable atmosphere which is beneficial to cure illness will be created. Hippocrates, an ancient Greek Philosopher once said, "As for the doctors, knowing a patient is more important than knowing what illness of the patient is". Thus, Traditional Chinese Medicine requires that during the course of disease treatment, on the one hand, physicians should note that there are differences in the shape of Qi and Blood, and have a comprehensive command of numerous factors, like patient's gender, age, constitution, psychology, locality and environment, etc. so as to implement "dialectical treatments" and "treatment in accordance with patient's individuality". On the other hand, they should also stay skeptical when doing medical inspection and think it over.

\section{Medicine as humane art}

Early in Jin Dynasty, Yang Quan stated that a doctor who can be entrusted must be a person of benevolence, intelligence and integrity. Pei Yizhong from Ming Dynasty also stressed in Speech on Physicians, "If you learn little about the ancient and contemporary, your knowledge is not abundant enough to comprehend Buddha nature, your talent does not stand out, and your heart is far from mercy, you'd rather make a living by farming or weaving than practicing medicine to ruin others". Chen Shigong laid down the rules in Five Precepts and Ten Disciplines, "Whenever see anyone in trouble, you should give him a hand according to your own ability. This can be called humane art".

There are many reasons for the conflicts between doctors and patients having come up in recent years: flaws in medical system, exaggerations of media as well as patients' misunderstanding towards life and death. But there is no denying that the direct cause is the lack of medical humanistic spirit. Trudeau is an American physician, whose epitaph wrote, "To cure sometime, to relieve often, and to comfort always". Nowadays, quite a few physicians only pay attention to human's biological properties but overlook their social properties and emotional properties. Due to the limitations of medicine, it is impossible to cure every single patient. As a physi- cian, his sacred duty is to spare no effort to help the sick, relieve their pain and comfort them as much as possible. In Lingshu, an excellent comment was made on talk therapy, "It is human nature to long for life rather than death. If we warn them what is harmful, remind them what is favorable and advise them what measures to take, exhort them with the pain of disease, how would they refuse to listen even though there are some unreasonable persons"? At one time, I have cured a female diabetic patient. During the process of treatment, various hypoglycemic agents lost efficacy owing to her depression, with the blood glucose reaching an abnormally high level. But after heart to heart communication with her, the amount of blood glucose was lowered down and under control, which has surpassed the effect of drugs.

\section{Benevolence prior to interest}

There are plenty of idioms, like "Practicing medicine to help the people", "genering hospiting" (Often used to praise a doctor with excellent medical skills), "Cure sickness with orange leaves and water from well", coming from allusions related to ancient Chinese physicians rescuing the dying and healing the wounded, which also symbolize their virtue "Putting benevolence prior to personal interest".

Zhang Zhongjing of the Eastern Han dynasty despised those vulgar people, who were only indulged in "chasing vanity and power", "obsessed with fame and fortune". Sun Simiao devoted himself to helping and saving others but expected nothing in return in his lifetime. Sun Simiao stated in Virtue of Great Physician, the first volume of Invaluable Prescriptions for Emergencies, "if someone suffering from sickness turns to you for help, you ought to treat them equally without discrimination, just like they are close to you, regardless of their social status, wealth, age, gender, intimacy (with you), nationality or intelligence". Moreover, he urged that physicians should not just seek personal interests by virtue of their strength in medical practice. Instead, physicians should sympathize with the sick, cure them without greed and realize how blessed they are. Additionally, they were not allowed to show off their medical ability by prescribing the rich with precious and rare medicine, making it hard to obtain, which went against the principle of benevolence and loyalty. Gong Tingxian admonished later generations that on the one hand, patients ought to revere rather than contempt doc- 
tors since they have sent for a doctor and entrusted him with their lives. On the other hand, doctors should not hesitate to treat with all sincerity because wealth is not the only criteria but if they do not take it serious, patients' lives will be put at risk. Chen Shigong drew up a set of rules in Five Precepts and Ten Disciplines, "however old or young, poor or wealthy the patients are, as long as they send for you, just go and visit. Don't delay or reject".

In Xu Lingtai's Complete Medical Book, written by Xu Dachun, also called Xu Lingtai, it suggests that medical practitioners should not become a charlatan, who "differentiates himself with strange prescriptions, or misleads others with unusual drugs, or flatters the rich with ginsengs or other restoratives, or fools the fumbler with alleged drugs prescribed by Buddha, or shocks the world with ridiculous fallacies, or deceives people with fabricated scriptures, or wants to look amazing by confusing the disease with another". It inspires the later doctors to cultivate enterprising initiative and sense of responsibility. Otherwise, they will only be regarded as physicians without virtue and end up with a tattered reputation.

\section{Respecting peers}

A doctor should respect their peers and counterparts. Sun Simiao declared that physicians should not laugh at, make improper comments on other peers' medical methods or gossip in public, discuss about them, flaunt their own fame, speak ill of other medical practitioners, or boast their own strength. He also lashed some people "become self-conceived after curing a disease occasionally, thinking no one can compare to him. Such physicians are incurable." To be an excellent doctor needs to "gather and accumulate knowledge from those wise and virtuous persons", remain humble and prudent all the time, respect medical peers, explore medical principles extensively and adopt useful advice from others. As a surgery specialist, Chen Shigong stated in Orthodox Manual of External Diseases, "do not contempt or insult your fellow townsman or peers of the same occupation. Remember to stay modest and thoughtful when getting along with people, in other words, respect the elderly, learn from the informed, forbear the arrogant, recommend the inferior In this way, there won't be any slanders or complaints but adherence to trust and peace".

Also in Curative Measures for All Diseases, Gong Tingxian sharply criticized those doctors who showed disrespect for medical peers. He made a point that those lacking virtues always tend to boast about his own strength but talk about others' weakness. When seeing the patients, they do not ask about the disease but only exaggerate the faults of the former doctor to scare them". Nowadays, it is one of the major reasons that lead to medical disputes. As you can often see in the outpatient clinic, when a patient finally goes to the hospital after long struggle, some doctors just say something negative, for example, "It is too late. You were wrongly diagnosed", "You took the wrong medicine." Hearing this, patients are scared to death and the illness gets worse. As a result, the conflicts between doctors and patients come along. Under such circumstances, as a doctor, the first thing to do is to make a careful analysis on the patient's condition, make it clear of the pros and cons of previous treatment so as to put it right, either misdiagnosis or mistreatment and determine what is the most effective medicines and measures to take for the patient next. Today's intensification of doctors-patients conflicts, increasing occurrence of medical disputes are closely related to counteractions among the medical peers. If only every single of us can inherit traditional medical virtues, a friendly and harmonious medical environment could be built, and medical disputes caused by "exaggerating the faults of the former doctor" could be avoided.

In conclusion, to reproduce the modern value of medical humanistic spirit, it is advisable to absorb humanistic spirit from Traditional Chinese Medicine and carry it forward by relieving the tensions between doctors and patients and improving the quality of medical services.

\section{Conflict of interest statement}

The authors have no conflict of interest to disclose.

\section{REFERENCES}

1. Zhang XQ. The absence of humanities and its return in medical education. Basic Clin Med 2012; 32(11): 1252-5.

2. Zhang $X Q$. Cognitive differences in the tension of doctor-patient relationship. China Hospital CEO 2013; (11):64-5

3. Zhou ZF. Comments on Zhouyi. Beijing: Zhonghua Book Company; 1991. p. 81.

4. Wang QQ. Selected readings from Neijing. Beijing: China Press of Traditional Chinese Medicine; 2007. p. 32. 